\title{
Substance use disorders among mentally ill patients in a General Hospital in Sri Lanka: prevalence and correlates
}

\author{
A Hapangama ${ }^{1}$, K A L A Kuruppuarachchi ${ }^{1}$, A Pathmeswaran ${ }^{2}$ \\ (Index words: mental illness, dual diagnosis, comorbidity, prevalence)
}

\begin{abstract}
Objectives To determine the prevalence, and demographic and clinical correlates of substance use disorders among the mentally ill.

Design and Setting Cross sectional analytical study. Data were collected from consecutive patients treated at a Tertiary care Psychiatry Unit, Western Province, Sri Lanka using an interview schedule administered by a senior registrar in psychiatry.

Measurements Diagnosis was based on ICD 10 criteria and the Clinician Rating Scale: Alcohol Use Scale (AUS) and the Drug Use Scale (DUS). The data were analysed using SPSS and WinPepi software programmes, utilising the chi squared, $t$ test and median test.

Results Among a sample of 325 patients, $66 \%$ were male; $33 \%$ were diagnosed with depressive disorder, $30 \%$ with schizophrenia, $23 \%$ with bipolar affective disorder and $13 \%$ with other disorders. Prevalence of comorbid substance use disorders was 43\% $(95 \% \mathrm{Cl}$ $38-49 \%)$. Alcohol was the commonest type of substance used. Those who had a comorbid substance use diagnosis were more likely to be male, have a lower educational level, be unemployed and have a poorer level of social support. They were also found to have poorer treatment adherence, increased number of hospital admissions and an increased association with episodes of violence.
\end{abstract}

Conclusion A significant proportion of patients were found to have a comorbid substance use diagnosis and associated complications. These findings have important implications for service provision for mentally ill patients with a comorbid substance use disorder.

Ceylon Medical Journal 2013; 58: 111-115

\section{Introduction}

Over the last few decades, there has been an increased recognition in Western countries of the co-occurrence of psychiatric illnesses with alcohol and drug use disorders and its consequences. Various terms such as dual diagnosis, dual disorders and comorbidity are used to denote this co-occurrence. In this article, we will be using the term comorbidity to describe the co-occurrence of a psychiatric illness with alcohol and drug use disorders.

Mental illnesses are reported to be associated with an increase in co-occurring substance use disorders compared to the general population [1]. For example, in one large general population survey conducted in the USA, the rate of lifetime alcohol or drug use disorder in the general population was approximately $17 \%$, compared to 47\%, 56\% and 30\% for people with schizophrenia, bipolar disorder, and mood or anxiety disorders respectively [2].

Comorbidity is associated with higher rates of specific negative outcomes such as severe financial problems from poor money management; unstable housing and homelessness; medication noncompliance, relapse and re-hospitalization; violence, legal problems, and incarceration; depression and suicide; family burden and high rates of sexually transmitted diseases [3]. Prospective studies have also shown that treatment outcomes are worse and these patients incur higher treatment costs due to increased utilisation of hospital and emergency services than those with only one disorder $[4,5]$.

Substance use in the mentally ill is frequently undetected and thus unaddressed [6]. During the last few years novel diagnostic and screening approaches have been developed in Western countries to detect substance use in these patients [7]. However, despite such developments in other countries, data regarding the prevalence, demographic factors and clinical correlates of patients with such comorbidity in Sri Lanka is sparse. Hence, the objectives of our study were to determine the proportion of such patients among patients with mental illnesses at a tertiary care hospital in the Western Province, Sri Lanka and to describe some correlates of comorbidity such as demographics and illness - related factors for substance use among the above group.

\section{Methods}

We carried out a cross sectional analytical study, at a tertiary care psychiatry unit in the Western Province, Sri Lanka. Data were collected from consecutive patients seen

Departments of ${ }^{1}$ Psychiatry and ${ }^{2}$ Public Health, Faculty of Medicine, University of Kelaniya, Ragama, Sri Lanka.

Correspondence: AH, e-mail: <ahapangama@yahoo.co.uk>. Received 24 December 2012 and revised version accepted 27 March 2013. Competing interests: none declared. 
at the unit from 1 January 2008 to 31 March 2009, using an interview schedule administered by a senior registrar in psychiatric. The primary outcome variable of interest was comorbidity. This variable was constructed using the diagnostic checklist filled out by the interviewer using the International Classification of Disease 10th version (ICD 10) as well as the Clinician Rating Scale: Alcohol Use Scale (AUS) and the Drug Use Scale (DUS) [8, 9]. In the Clinician Rating Scale: Alcohol Use Scale (AUS) and the Drug Use Scale (DUS), clinicians rate on a 5-point scale the extent of alcohol, cannabis, and cocaine abuse over the past 6 months. Points 1 and 2 correspond to no substance use and substance use without abuse, respectively, and points 3,4 , and 5 correspond to substance abuse, dependence, and severe dependence, respectively.

The study focussed on all consecutive in and outpatients above the age of 18 years who were able to give written informed consent.

Patients who fulfilled ICD 10 criteria for a mental disorder and a substance use disorder, as well as patients who fulfilled ICD 10 criteria only for a mental disorder but had Clinician Rating Scale: (AUS) and (DUS) criteria for a substance use disorder were included in to the group of "patients with comorbidity". Patients who fulfilled ICD 10 criteria for a mental disorder but who did not fulfil ICD 10 or Clinician Rating Scale: (AUS) and (DUS) criteria for a substance use disorder were included in the group of "those without comorbidity". Patients who had only a substance use disorder according either ICD 10 or Clinician Rating Scale: (AUS) and (DUS) without an ICD 10 mental disorder were excluded from the study.

\section{Sample size}

Since studies done elsewhere have shown prevalence of comorbidity of $20-50 \%$, we assumed a prevalence of $30 \%$. For an estimate to be within $5 \%$ of the actual value, the required sample size was 325 . We used the following criteria to define some of the demographic and clinical variables. A person was considered to have social support if there was at least one regular caregiver whom the patient could nominate. Patients were categorised as having poor adherence to medication if he/she had ceased medication for more than two days for any reason, without medical advice, during the time he/she was treated for the mental illness. A patient was categorised as being violent if episodes of physical aggression to others were noted in his/her records or if patient informed of such incidents to the interviewer. Recurrences of symptoms following the initial episode as per the ICD 10 criteria were defined as relapses.

Data were analysed using the SPSS and WinPepi software programmes. Chi-squared tests for categorical data, t- tests for continuous variables and the median test in situations where the use of $t$ test was inappropriate were used for univariate analysis. Approval was obtained from the Ethics Review Committee of Faculty of Medicine,
University of Kelaniya. Approval was taken from the authors for the use of Clinician Rating Scale: (AUS) and (DUS).

\section{Results}

The total sample size was 325. Of the study population $64.6 \%$ was from the out-patient setting (Table 1 ). The mean age of the study group was 36 years. Of the study population $66.2 \%$ were male, $51.7 \%$ of the participants were married and $39.7 \%$ had an education up to GCE O/L. One hundred and nine (33.5\%) of the study group had depressive disorders while 97 (29.8\%) had schizophrenia and 76 (23.4\%) had bipolar affective disorder (Table 2).

Table 1. Study group characteristics

\begin{tabular}{lc}
\hline Characteristic & Number $(\%) n=325$ \\
\hline In patients & $115(35.4)$ \\
Outpatients & $210(64.6)$ \\
Gender - Male & $215(66.2)$ \\
\multicolumn{1}{c}{ Female } & $110(33.8)$ \\
Educational level $-\quad$ Up to O/L & $129(39.7)$ \\
$\quad$ More than O/L & $196(60.3)$ \\
Marital status - Married & $168(51.7)$ \\
$\quad$ Never married & $141(43.4)$ \\
$\quad$ Separated/ divorced & $16(4.9)$ \\
\hline
\end{tabular}

One hundred and thirty one patients fulfilled ICD 10 criteria for substance use disorders. A further 10 patients fulfilled Clinician Rating Scale: (AUS) and (DUS) criteria. The total with comorbid substance use disorders was 141 . The prevalence of substance use disorders in the study population was $43.4 \%$ (95\% CI 38.1\%- 48.8\%). There were 140 (65.1\%) males and 1 (0.9\%) female among those with comorbidity. The prevalence of comorbidity among the male population was $43.1 \%$ (95\% CI 37.8\%- $48.5 \%$ ). The mean ages were 34.5 years for those with comorbidity and 37.0 years for those without comorbidity $(\mathrm{t}=1.605,>0.05$, 95\% CI -0.55 - 5.44).

Demographic and clinical correlates of substance use among the two groups are described in Table 3. Of the patients with comorbidity 58.9\% claimed they used substances to alleviate boredom while $41.8 \%$ said they used substances to improve their attention and $21.9 \%$ to reduce symptoms of the mental illness.

Of the patients $10.8 \%$ were found to have substance use disorders due to the use of two or more substances while $20.6 \%$ had comorbid alcohol use disorders only (Table 4). The female participant with comorbidity had an alcohol use disorder. Among male participants with comorbidity the prevalence was alcohol $30.7 \%$, tobacco $27.4 \%$ and cannabis use disorder $17.7 \%$. Comorbidity 
among those with bipolar affective disorder was 57.8\% while it was $44.3 \%$ for schizophrenia and $32.4 \%$ depressive disorders.

Among patients with bipolar affective disorder, 23.7\%, had comorbidity due to alcohol, 21.1\% for tobacco and
$7.9 \%$ for cannabis respectively (Table 2). In patients with depressive disorders comorbidity with alcohol $21.1 \%$, tobacco $9.2 \%$ and cannabis $1.8 \%$. In patients with schizophrenia comorbidity was alcohol $11.3 \%$, tobacco $23.7 \%$ and cannabis $19.6 \%$ (Table 2).

Table 2. Prevalence of substance use disorders according to diagnostic category

\begin{tabular}{|c|c|c|c|c|c|}
\hline Diagnosis & $\begin{array}{c}\text { Number } \\
(\%)\end{array}$ & $\begin{array}{c}\text { Alcohol use } \\
\text { disorders } \\
\%(95 \% \text { CI) }\end{array}$ & $\begin{array}{c}\text { Tobacco use } \\
\text { disorders } \\
\%(95 \% \text { CI) }\end{array}$ & $\begin{array}{c}\text { Cannabis use } \\
\text { disorders } \\
\%(95 \% \quad C I)\end{array}$ & $\begin{array}{c}\text { Betel use } \\
\text { disorders } \\
\%(95 \% \quad C I)\end{array}$ \\
\hline Depressive disorder & 109 (33.5) & $21.1(14.2-29.5)$ & $9.2(4.7-15.7)$ & $1.8(0.3-5.9)$ & $6.4(2.8-12.3)$ \\
\hline Schizophrenia & $97(29.8)$ & $11.3(6.1-18.9)$ & $23.7(16.1-32.9)$ & $19.6(12.6-28.4)$ & $9.3(4.6-16.3)$ \\
\hline Bipolar affective disorder & $76(23.4)$ & $23.7(15.2-34.2)$ & $21.1(12.9-31.3)$ & $7.9(3.2-15.7)$ & $5.3(1.7-12.2)$ \\
\hline Delusional disorder & $20(6.1)$ & $45.00(24.6-66.7)$ & $25.00(9.7-47.0)$ & $5.00(0.2-22.3)$ & $5.00(0.2-22.3)$ \\
\hline Others & $23(7.1)$ & $26.1(11.3-46.6)$ & 21.7 (8.4-41.8) & $4.3(0.2-19.6)$ & $13.0(3.4-31.5)$ \\
\hline
\end{tabular}

Table 3. Demographic and clinical correlates of substance use

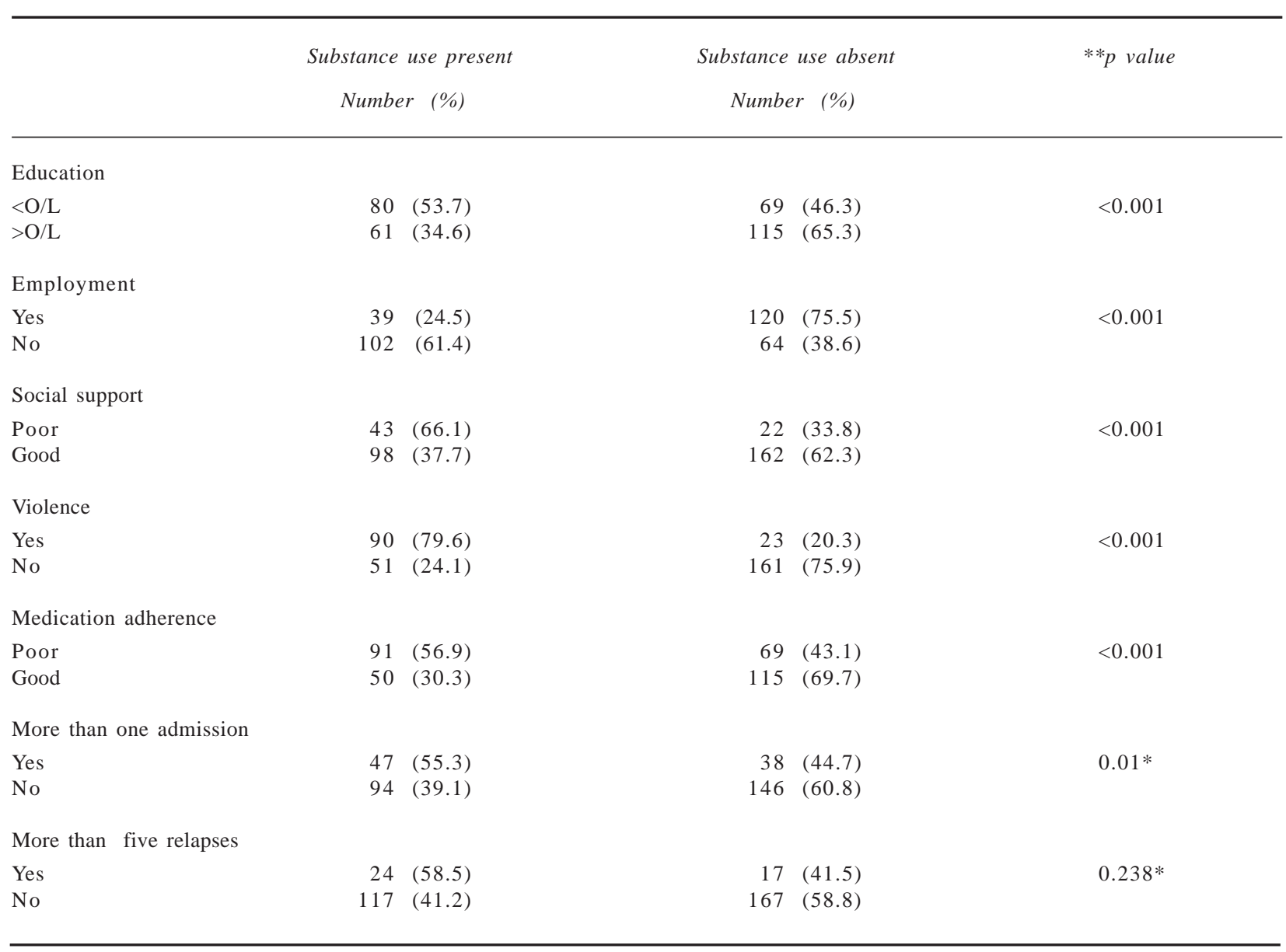

\footnotetext{
${ }^{* *} p$ values are based on the chi squared test unless indicated by an asterisk $\left({ }^{*}\right)$ where the $p$ values are based on the median test
} 
Table 4. Substance use disorders in the study population

\begin{tabular}{lc}
\hline Substance & \# Number (\%) \\
\hline Alcohol use disorders & $67(20.6)$ \\
Tobacco use disorders & $59(18.2)$ \\
Cannabis use disorders & $38(11.7)$ \\
Betel use disorders & $24(7.4)$ \\
Opioid use disorders & $4(1.2)$ \\
Multiple substance use disorders & $35(10.8)$ \\
\hline
\end{tabular}

\# Study participants may fall in to one or more substance use disorders depending on the number of substances they misuse or are dependent; therefore the total number and total percentage would exceed 141 and $100 \%$ respectively.

\section{Discussion}

Other authors have highlighted the significant underdiagnosis of substance use disorders, when only a single set of diagnostic criteria are being used [10]. To minimise this error, we used two sets of diagnostic criteria (ICD 10 and Clinician Rating Scale: (AUS) and (DUS)) and for diagnosis of substance use disorder participants had to fulfill only one set of criteria. Of the study population 43.4\% was diagnosed to have a comorbid substance use disorder. Prevalence of comorbidity in other parts of the world shows figures between $20-45 \%$ [2, 11, 12$]$.

We found that patients who are male, of younger age and a lower educational level have an increased association with comorbidity while female gender has a lower association. Similar findings have been reported in other countries as well $[13,14]$. Of females in our study population 99\% did not have comorbid substance use disorders. This may be attributed to socio-cultural and religious influences prevalent in Sri Lanka [15].

We found that patients with comorbidity had more admissions, lesser compliance with treatment and a higher number of incidents of violence, a finding reported in other studies as well [4]. There was no significant difference between the two groups in the number of relapses. However, research done elsewhere have found that patients with comorbidity tend to have more relapses than those without comorbidity [4]. Our inability to verify information from a second source due to ethical and practical issues may have contributed to this difference.

Similar to other studies we found reduction of boredom, improvement of attention and alleviations of symptoms of mental illnesses as the most common reasons for substance use [16]. The Epidemiological Catchment Area study had found that $47 \%$ of patients with schizophrenia and 56\% with bipolar affective disorder had comorbidity [3]. Our figures for the above disorders were $44 \%$ and $57 \%$ respectively.
Nationwide prevalence studies on alcohol and drug use disorders among the general population are limited in Sri Lanka and it is difficult to compare the existing data due to differences in the criteria used to detect substance use. However, in a recent survey conducted in Sri Lanka, prevalence of current alcohol use was $35.6 \%$ and current smoking 33.2\% [17]. In this survey current users have been defined as those who have used the given substance within the previous month, whereas in our study participants had to fulfill several criteria either during the preceding one year (ICD 10) or six months Clinician Rating Scale: (AUS), (DUS). In our sample, alcohol (20.6\%) was the single commonest substance leading to a comorbid diagnosis followed by disorders due to tobacco (18.2\%) and cannabis (11.7\%). A study done in a Western country had reported alcohol, cannabis and cocaine respectively as the commonest substances used by patients with mental illnesses [18]. These differences probably highlight the variation in availability and affordability of substances in Sri Lanka.

A study has reported the prevalence of comorbid alcohol, tobacco and cannabis use disorders among males in Sydney as $15.4 \%, 48 \%$ and $14.4 \%$ respectively [19]. Our figures were substances for the male were alcohol $30.1 \%$, tobacco $27.4 \%$ and connabis $17.6 \%$. Similar to studies done elsewhere alcohol was the commonest substance used by patients with bipolar affective disorder [19]. In our sample tobacco use disorders were the commonest substance use diagnosis among patients with schizophrenias with a prevalence of $23.7 \%$, however there are reported figures as high as $79 \%$ among patients with schizophrenia [20].

Our study has several limitations. We could not use biochemical tests to verify the substance use. The study sample consisted mainly of urban and semi-urban dwellers. Their demographic characteristics and prevalence of substance use may be different from patients from rural areas. Prescription medications such as benzodiazepines which can be abused have not been included in this study. The study consisted mainly of patients with severe mental illnesses such as schizophrenia, bipolar affective disorder and depressive disorders. We have not considered the severity of mental illness and the presence of personality disorders; however these factors may act as confounders for substance use, poor compliance and relapse rate. We have used pooled data of several different substances to describe the correlates however some substances may have a lesser association with certain clinical correlates than others (e.g. betel with violence).

Our findings have several important implications. A considerable proportion of mentally ill patients were found to have comorbid substance use disorders and associated complications. Patients with comorbidity were more likely to be of male gender, younger age, lower educational level, poorer level of social support and to be unemployed. 
Patients with comorbid substance use disorders were also found to have increased number of hospitalisations, poorer adherence to medications and higher rates of violence.

\section{Acknowledgements}

We would like to thank Professor Kim T. Mueser, Professor of Psychiatry, Dartmouth Medical School, Hanover, New Hampshire, USA for granting us permission to use the Clinician Rating Scale:Alcohol Use Scale (AUS) and the Drug Use Scale (DUS).

\section{References}

1. Kessler RC, Crum RM, Warner LA, et al. Lifetime cooccurrence of DSM-III-R alcohol abuse and dependence with other psychiatric disorders in the National Comorbidity Survey. Archives of General Psychiatry 1997; 54: 313-21.

2. Regier DA, Farmer ME, Rae DS, et al. Comorbidity of mental disorders with alcohol and other drug abuse. Results from the Epidemiologic Catchment Area (ECA) study. Journal of the American Medical Association 1990; 264: 2511-8.

3. Drake RE, Mueser KT. Psychosocial Approaches to Dual Diagnosis. Schizophrenia Bulletin 2000; 26: 105-18.

4. el-Guebaly N. Concurrent substance-related disorders and mental illness: the North American experience. World Psychiatry 2004; 3: 182-7.

5. U.S. Department of Health and Human Services. Substance Abuse and Mental Health Services Administration. Report to Congress on the prevention and treatment of co-occurring substance abuse disorders and mental disorders. U.S. Department of Health and Human Services. Rockville, Md.USA, 2002.

6. Cantwell R, Harrison G. Substance Misuse in the Severely Mentally Ill. Advances in Psychiatric Treatment 1996; 2: 117-24.

7. Rosenberg SD, Drake RE, Wolford GL, et al. The Dartmouth Assessment of Lifestyle Instrument (DALI): A substance use disorder screen for people with severe mental illness. American Journal of Psychiatry1998; 155: 232-8.

8. World Health Organization. The ICD-10 classification of mental and behavioural disorders. Clinical descriptions and diagnostic guidelines. Tenth Revision. Geneva: World Health Organization; 1992.

9. Drake RE, Mueser KT, McHugo GJ. Clinician Rating Scales: Alcohol Use Scale (AUS), Drug Use Scale (DUS), and Substance Abuse Treatment Scale (SATS). In: Sederer LI,
Dickey B, eds.Outcomes Assessment in Clinical Practice. Edited by Sederer LI, Dickey B. Baltimore: Williams and Wilkins, 1996: 113-6.

10. Morojele NK, Saban A, Seedat S. Clinical presentations and diagnostic issues in dual diagnosis disorders. Current Opinion in Psychiatry 2012; 25: 181-6.

11. Weaver T, Madden P, Charles V, et al. Comorbidity of Substance Misuse and Mental Illness Collaborative study team. Comorbidity of substance misuse and mental illness in community mental health and substance misuse services. British Journal of Psychiatry 2003; 183: 304-13.

12. Menezes PR, Johnson S, Thornicroft G, et al. Drug and alcohol problems among individuals with severe mental illness in South London. British Journal of Psychiatry 1996; 168: $612-9$.

13. Mueser KT, BennettM, Kushner MG. Epidemiology of substance use disorders among persons with chronic mental illnesses. In: Lehman, AF, and Dixon, L, eds. Double Jeopardy: Chronic Mental Illness and Substance Abuse. New York: Harwood Academic Publishers, 1995a: 9-25.

14. Rabinowitz J, Mark M, Popper M, Slyuzberg M. Reported comorbidity of mental disorders with substance abuse among psychiatric inpatients in Israel. The Journal of Behavioural Health Services and Research 1996; 23: 471-8.

15. Rodrigo C, Welgama S, GunawardanaA, et al. A retrospective analysis of cannabis use in a cohort of mentally ill patients in Sri Lanka and its implications on policy development. Substance Abuse Treatment, Prevention, and Policy 2010; 5: 16.

16. Mueser KT, Drake RE, Wallach MA. Dual diagnosis: a review of etiological theories. Addictive Behaviours 1998; 23: 717-34.

17. Alcohol and Drug Information Centre. Spot Survey. Research and Evaluation Programme. Colombo: Alcohol and Drug Information Centre; 2012.

18. Mueser KT, Yarnold PR, Levinson DF, et al. Prevalence of substance misuse in schizophrenia: Demographic and clinical correlates. Schizophrenia Bulletin 1990; 16: 31-56.

19. Hermens DF, Scott EM, White D, et al. Frequent alcohol, nicotine or cannabis use is common in young persons presenting for mental healthcare: a cross-sectional study. British Medical Journal Open 2013; 3: e002229.

20. Zhang XY, Liang J, Chen DC, et al. Cigarette Smoking in Male Patients with Chronic Schizophrenia in a Chinese Population: Prevalence and Relationship to Clinical Phenotypes. Public Library of Science ONE 2012; 7: e30937. 